\title{
SENSORY TRANSDUCTION AND SUBJECTIVE EXPERIENCE: EXPRESSION OF EIGHT GENES IN THREE SENSES SugGESTS A RADICAL MODEL OF CONSCIOUSNESS
}

\author{
Chris King \\ Mathematics Department, University of Auckland, Auckland, New Zealand \\ Received March 15, 2009; accepted March 25, 2009
}

\begin{abstract}
Recent research into whole genome mapping of the mouse brain has made possible direct investigation of the brain expression of unusual genes. A search of the Allen Brain Atlas database has provided genetic and neuro-anatomical evidence for widespread specific expression in the brain of eight genes specific to sensory transduction, in vision, hearing and touch. A novel biophysical model is proposed for the function of these proteins, in generating the internal model of experiential reality.
\end{abstract}

Key words: Brain; Consciousness; Free-Will; Genes; Quantum Excitations

\section{INTRODUCTION}

Recent research in whole genome mapping of the mouse brain (Lein et al., 2007) has made it possible to investigate the potential central nervous function of genes that might otherwise be associated primarily with peripheral sensory transduction. At the same time, the actual molecules involved in sense transduction, in vision, hearing and touch are being characterized. The first putative transduction molecule for mammalian touch, stomatin-like protein 3 (SLP3, or Stoml3) was reported in 2007 (Wetzel et al., 2007) and putative molecules in the auditory transduction pathway, epsin (Parkinson \& Brown, 2002; Di Palma et al., 2001) and cadherin 23 (otocadherin) (Di Palma et al., 2001) have only been reported in the last five years and otoferlin in 2006 (Parsons, 2006; Roux et al., 2006). Research into the genetic evolution of the visual system has also unearthed provocative new findings about vision, which became the trigger for this hypothesis. In parallel with the usual cilia-based photo-transducer molecule c-opsin are retinal ganglion cells, which use melanopsin, or ropsin related to insect opsins (based on organelles called rhabdomeres), which depolarize rather than hyperpolarize (Fernald, 2006). It has also been discovered that both types of opsin work in opposition in the reptile parietal (pineal) eye (Su et al., 2006).

\section{INVESTIGATION}

Interest in such idiosyncratic incidences of sensory genes became the stimulus for making a short investiga tion of molecules associated with sensory transduction in brain tissues, using the Allen Brain Atlas (Lein et al., 2007) of the mouse. This immediately threw up a further opsin variant, encephalopsin (Blackshaw \& Snyder, 1999), discovered in 1999 and known to have a broad and selective distribution in the brain, including, but not restricted to, areas involved in visual processing. At the same time as making this search, Nature reported the discovery of Stoml3 in touch transduction (Wetzel et al., 2007) and a search revealed this also has a wide brain distribution. Stoml 3 was found to bind specifically to acid-sensitive ion channels ASIC2 and 3 and a search likewise found a CNS-wide expression of these genes. Finally a search was made for auditory molecules, which threw up epsin and cadherin-23 (Parkinson \& Brown, 2002; Di Palma et al., 2001), which likewise show brain-wide specific expression. Subsequently, the recent characterization of otoferlin (Parsons, 2006; Roux et al., 2006), claimed to be key to the sensitivity of auditory transduction led to exploration of this auditory molecule as well, providing evidence of widespread expression from five genes involving three senses.

\footnotetext{
* Correspondence to: Chriss King, University of Auckland, e-mail:king@math.auckland.ac.nz
} 
In support of the central nervous expression of genes believed to be associated primarily with sensory transduction, an exploration of: (a) rhodopsin, and encephalopsin, (b) otocadherin, espin, and otoferlin and (c) acid-sensitive ion channels ASIC2 and 3 and stomatin-like protein 3 using the Allen Brain Atlas is included in the figures. Figure 1A shows lateral sagittal views of the whole mouse brain for five of these genes, supporting their expression in the brain. Figure 1B looks in detail at an area of the ventral lateral cortex illustrating similar expression of each of these genes at the cellular level. Figure 1C shows the specific expression of rhodopsin in the cortex focused in areas consistent with visual function. Figure 1D exemplifies more specialized activity of two of them in the olfactory bulbs and cerebellum. Figure $1 \mathrm{E}$ shows varying expression for four of the genes in the parietal cortex.

\section{Could the CNS Contain Transduction Cascades?}

Opsins are clearly transducers from photonic to electrochemical (Blackshaw \& Snyder, 1999). Encephalopsin is also expressed in other organs, and is also referred to as panopsin, so could have another generalized cellular function. However there are several other opsins of interest expressed in the CNS. Pinopsin is not confined to the pineal but also occurs widely in the brain. In addition vertebrate ancient opsin is also expressed in regions bordering the pineal. Rhodopsin has activity concentrated in individual neurons across the cortex with a specific focus in the occipital, consistent with a function in the primary visual cortex (Blackshaw \& Snyder, 1999).

Otoferlin, which was only characterized in Oct 2006, is as close as research can establish to the transduction step. Otoferlin functions right in the critical steps of the signaling cascade stimulating the fast kinetics of the most mature $\mathrm{Ca}$ dependent neurotransmitter vesicles, thus triggering the receptor cell response, and it's also transmembrane and possibly a $\mathrm{Ca}$ channel so it is right on the transduction interface (Roux et al., 2006). In particular Parsons 6 notes that the hair cell has evolved a unique calcium-sensing molecule, otoferlin, for controlling neurotransmitter release. The action of otoferlin allows a hair cell's specialized synapses - ribbon synapses, a specific class of afferent synapse common to sensory systems - to meet the requirements of hearing. Roux et. al. (2007) describes otoferlin as a novel protein and transmembrane cochlear-expressed gene. So its function looks like a $\mathrm{Ca}++$ ion channel or channel modulator that excites mature kinetically unstable vesicles. This could be the direct result of a phononic or solitonic event in the membrane.

The presence of no less than three molecules from the auditory transduction pathway - otoferlin, otocadherin and espin in the CNS suggests functional linkage in the CNS and a possible signaling cascade. All three don't have to be directly involved in transduction, but all may be essential to it, as is evidenced by deafness studies (Roux et al., 2006).

SLP3 is a transduction modulator, which binds specifically to acid-sensitive ion channels ASIC 2\&3. The atlas found very similar cortical distributions of all three molecules, again setting up a putative model for a transduction cascade here as well. However ASIC may have more general ion-channel functions in the CNS which makes the role of SLP3 interesting. Wetzel et. al. (2007) show mechano-sensitive ion channels found in many sensory neurons do not function without SLP3 including touch mechanoreceptors as a whole and cites their coupling to ASIC $2 \& 3$.

\section{Subjective Consciousness and Biophysical Transduc- tion}

Interest in the biophysical basis of subjective consciousness has become central to the emerging area of consciousness research. A variety of models have been put forward for the involvement of CNS proteins in, quantum computation by orchestrated objective reduction in microtubules (Hameroff, 2006; Hagan, Hameroff, \& Tuszynski, 2002) and others involving coherent quantum excitations including a protein/water/EM field model (Georgie, Papaioanou, \& Glazebrook, 2004; Georgie \& Glazebrook, 2006, 2007). A variety of functional proteins in the CNS are under investigation to test for their possible role in the biophysical underpinning of subjective consciousness. It has also been proposed that conscious anticipation might be made possible through quantum excitations both emitted and absorbed by the CNS (King, 1997, 2006).

Although subjective consciousness has many attributes, from the sense of self-awareness (self-consciousness) through semantic and rational processes (rational mind) and working memory, some of which involve subliminal processing on the fringes of consciousness or unconsciously, there is a major central arena of conscious experience, sometimes referred to as the Cartesian theatre (Baars, 1997; Dennet, 1991), which gives the subjective expression of an envelope of sensory experience, whether it involves experiences of the external world or purely internal states such as dreaming. This in turn gives rise to the notorious binding problem - how a distributed parallel processing organ like the cortex with disparate sensory areas can bring in all back together Baars, 1997; Dennet, 1991). However the primacy of internal 'sensory experience' in subjective consciousness suggests a biophysical support based on the same principles as are involved in sensory transduction.

The occurrence of putative sensory transduction genes in the central nervous system is consistent with a novel biophysical model supporting subjective consciousness - that the distributed functioning of the central nervous system provides an 'internal sensory system' which 
can generate abstracted sensory experiences of reality forming an 'internal model of reality' using the same physical principles as are involved in sensory transduction in a bi-directional manner, enabling coherent generation and reception of biophysical excitations, particularly those associated with vision and audition. Olfaction has a fundamentally different basis, both in brain architecture and in the fact that it involves specific molecular receptors, which cannot regenerate their stimuli by reverse transduction, although there is evidence for olfactory synesthesia (Simner \& Ward, 2006). Some forms of synesthesia, such as responding with feeling to seeing another person's finger touched (Banissy \& Ward, 2007), may also involve specific interactive circuitry, including mirror neurons (Rizzolatti \& Craighero, 2004).

The model gives a succinct explanation of why subjective experience such, as dream, memory and reflection, as Carl Jung (1961) put it, so successfully evokes the deep qualitative differences between the senses, when a purely electrochemical model has no qualitative differences between the senses, except in terms of differential developmental and stimulus-induced processing connectivities. The wide distribution of each of these molecules, not confined to one sensory area, suggests that the evolution of the cortex as an adaptable system, has resulted in a flexible design, in which widespread areas of the brain may be capable of generating dynamics simulating more than one sensory process biophysically, consistent with descriptions of kaleidoscopic synesthesia in the medical literature, in psychedelic folklore (Simner \& Ward, 2006), and manifest in ancient cave art running far back into our human origins (Rudgley, 1993).

By contrast with all other areas of scientific discovery, from the human genome to cosmological grand unification, the nature and basis of conscious experience remains the principal scientific area in the third millennium for which there is yet no realizable candidate theory, nor even a qualitative understanding in prin- ciple, of how our 'internal model of reality' is generated. While consciousness research has come in from the cold as an accepted scientific research area (King, 2006) there are still major stumbling blocks to a realizable theory of consciousness, including the 'hard problem' (Chalmers, 1995) - whether subjective consciousness is in any way qualitatively identifiable with an objective description of reality, to the 'binding problem' - how multifarious processes come together to convey the impression of a 'Cartesian theatre' of the mind (Baars, 1997; Dennet, 1991).

Research into the biophysical basis of consciousness remains obscure, invoking a variety of speculative theories, few of which have convincing experimental support at the cellular level. Nevertheless subjective conscious states, from dreaming, through psychedelic states, to memory and imagination, each possess a veridical reality, which is of the same broad sensory nature as an external experience. Indeed dreaming can become all too real, by any sensory measure, despite attempts at lucidity checks!

Although we conceive of the nominal five senses - vision, touch, hearing taste and smell - as biological adaptions, they are actually manifestations of the principal quantum modes by which an organism can interact with the physical world. Vision is photon-orbital interaction, hearing comes somewhere between phonon-orbital and the mechano-receptor dynamics of touch, taste and smell are traditionally defined as an orbital-orbital shape-fitting, although some research 29 suggests smell involves quantum vibration modes as well. Sensory transduction is also capable of working at the quantum limit. Frog rod cells are capable of responding to single photons 26, pheromones likewise can elicit a response from a single molecule (especially in insects) and the limits of audition involve movements of the cochlear membrane of the order of a hydrogen atom radius (Rudgley, 1993; King, 2006).

\section{Figure 1.}

Figure 1A. Large scale mouse brain expression profiles of encephalopsin (Opn3), otocadherin (Cdh23), espin (Espnl), otoferlin (Otof) and Stom3 (Allen Brain Atlas) illustrate the wide and discretely specific expression of sensory transduction molecules for three senses, vision, hearing and touch in the central nervous system. Does this mean that the 'internal model of reality' evokes subjective experience using similar molecules to the physical senses?

Figure 1B. Exploded view in the lateral ventral cortex at the cellular level of expression of encephalopsin (Opn3), otocadherin (Cdh23), espin (Espnl), otoferlin (Otof) and Stom3 demonstrate specific expression of a similar type in cortical tissue at the cellular level.

Figure 1C. Expression of rhodopsin in the CNS shows both strong selective neuronal expression and a focal expression in the occipital cortex consistent with expression in the primary visual areas.

Figure 1D. Specialized expression of encephalopsin (Opn3) in the cerebellum, and of otocadherin (Cdh23) in the olfactory lobes illustrate divergent specialized function of these genes in specific brain areas contrasting with their similar expression in figure 2 .

Figure 1E. Expression of encephalopsin (Opn3), stoml3, otocadherin (Cdh23) and otoferlin (Otof) in the parietal cortex illustrate differing modes of cortical expression. 


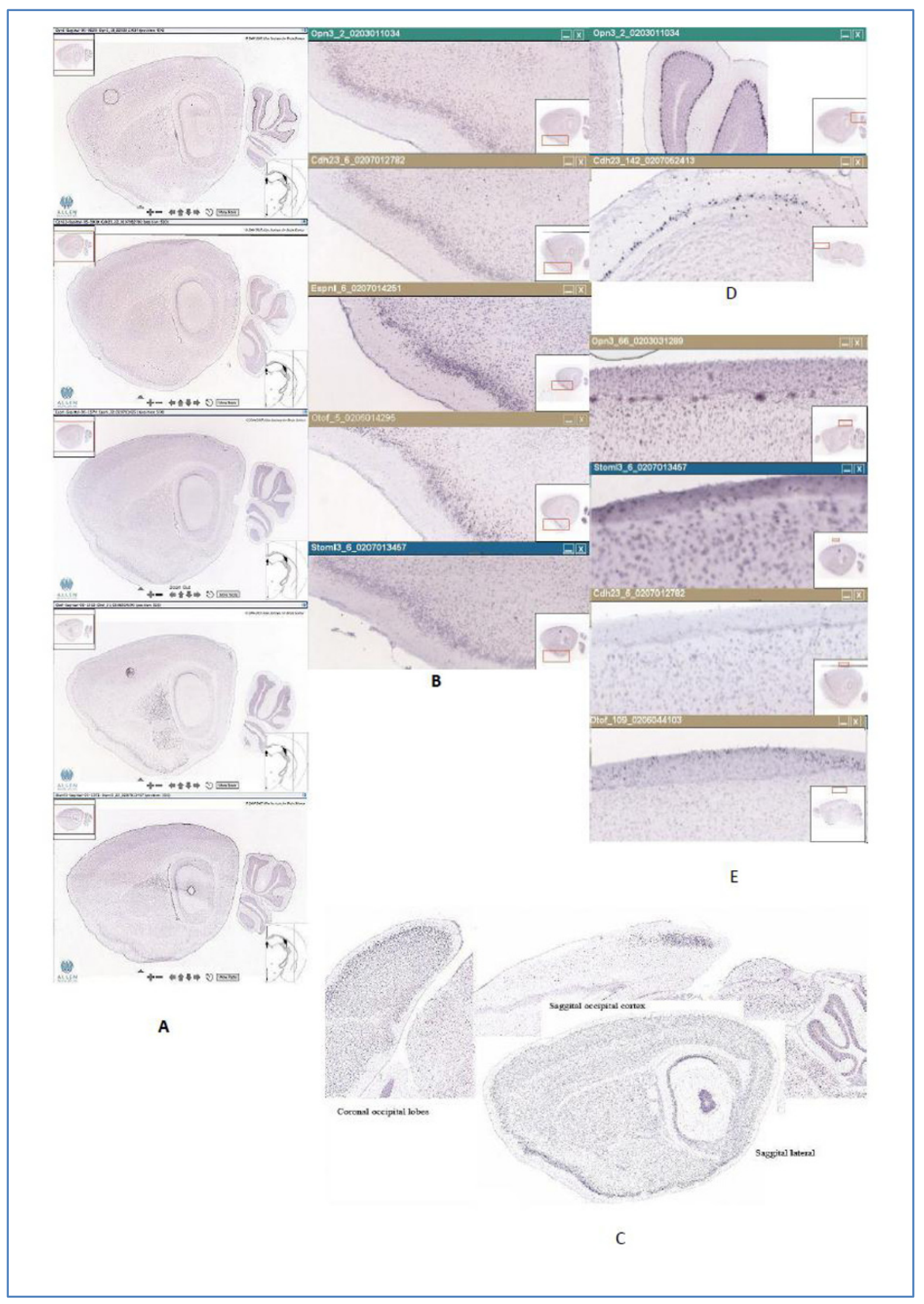




\section{CNS Gene Expression and REST}

One line of caution about interpreting actual function of genes expressed in the brain is that neuronal cells have relatively open transcription, due to the very low levels of REST, the zinc finger protein RE-1 silencing transcription factor in neuronal cells. The transcriptional repressor REST controls neuron-specific gene transcription via recruitment of histone deacetylases (Thiel, Lietzand, \& Hohl, 2004). "In neurons, only very low levels of REST can be detected. In fact, a decrease in the REST concentration during neuronal differentiation is most probably the essential prerequisite for transcription of neuronal genes. The chromatin configuration of neuronal genes is open, due to an extensive acetylation of the core histones. Transcriptional activators, as well as the RNA polymerase II complex, can gain access to the DNA and trigger gene transcription. In non-neuronal cells, however, REST is present and binds in a sequence-specific manner to several neuronal genes." (Thiel, Lietzand, \& Hohl, 2004)

The relatively open transcription of neuronal cells has led some researchers to suggest that transcription in neurons is not a reliable indicator of gene expression since the relaxation of REST allows for wholesale expression of neuronal-related genes, which may not be actually translated due to RNA processing after transcription. This however runs counter to the fact that neurons, participating in the most complex finely tuned organ of all - the brain need to have corresponding acuity of gene transcription. The idea that neurons are simply an open Pandora's box of randomly transcribed genes is not an accurate assessment.

However other evidence suggests that the influence of RNA may occur at a pre-transcriptional level and in a highly elegant manner that would permit just the sort of very specific gene expression required in the brain. Kuwabara et. al. (2004) describe a small dsRNA that activates the expression of neuron-specific genes at a transcriptional level and that alone is capable of inducing the differentiation of neuronal progenitor cells into neurons. This RNA may represent the first member of a group of small RNAs that directly modulate gene expression at the transcriptional level. Intriguingly, this RNA, which is expressed in the nucleus, rather than the cytoplasm where most miRNAs and siRNAs are found, neither inhibits expression of REST (not having sequence correspondence with REST mRNA), nor does it inhibit REST's binding to DNA transcription sites, but rather alters REST's activity, from binding to other repressors, such as CoREST to binding with activators of gene expression (Thiel, Lietzand, \& Hohl, 2004).

The purpose of the current paper is not to establish beyond doubt that sensory transduction molecules are functionally expressed in the brain, but simply to show that gene expression at the level of anatomical investigation is consistent with this idea. Several of the genes cited, particularly rhodopsin, appear to be functionally transcribed as their pattern of occurrence is anatomically specific and in the latter case, correlated with the visual cortex, suggesting that at least some of these candidates do have functional expression in the CNS.

\section{CONCLUSION}

While the evidence presented is from distributed gene expression and thus in no way confirms these molecules are performing a sensory transduction function in the central nervous system, the theory does present an innovative and scientifically provocative biophysical hypothesis about the genesis of the 'internal model', which could also have significant implications for cognitive science. Physically transduced quantum excitations phase correlated with the electrodynamics underlying the electroencephalogram could provide a realizable means for the brain to generate quantum entangled states, permitting forms of quantum computing using our massively parallel, phase coupled brain dynamics. Some models ((King, 1997, 2006) also suggest such processes could also have an anticipatory function which might help explain free-will.

\section{REFERENCES}

Baars, B. (1997). In the Theatre of Consciousness: Global Workspace Theory, A Rigorous Scientific Theory of Consciousness. Journal of Consciousness Studies, 4(4): 292-309.

Banissy, M. J., \& Ward, J. (2007). Mirror-touch synesthesia is linked with empathy. Nature Neuroscience, 10, 815-816.

Blackshaw, S, \& Snyder, S.H. (1999). Encephalopsin: A Novel Mammalian Extraretinal Opsin Discretely Localized in the Brain. The Journal of Neuroscience, 19, 3681-3690.

Chalmers, D. (1995). The Puzzle of Conscious Experience. Scientific American, 273,(Dec.) 80-6.

Dennet, D. (1991). Consciousness Explained. Little, Brown and Company.

Di Palma, F., Holme, R.H., Bryda, E.C., Belyantseva, I.A., Pellegrino, R., Kachar, B., Steel, K.P., Noben-Trauth, K. (2001). Mutations in Cdh23, encoding a new type of cadherin, cause stereocilia disorganization in waltzer, the mouse model for Usher syndrome type 1D. Nature Genetics, 27, 103-107.

Fernald, R. D. (2006). Casting a genetic light on the evolution of eyes. Science, 313, 1914-18.

Georgiev, D.D., Papaioanou, S.N, \& Glazebrook, J.F. (2004). Neuronic system inside neurons: molecular biology and biophysics of neuronal microtubules. Biomedical Reviews, 15, 67-75.

Georgiev, D.D., \& Glazebrook, J.F. (2006). Dissipationless waves for information transfer in neurobiology - some implications. Informatica, 30, 221-232.

Georgiev, D.D., \& Glazebrook, J.F. (2007). Conformational dynamics and thermal cones of C-terminal tubulin tails in neuronal microtubules. Neuroquantology, 5, 62-84.

Hagan, S., Hameroff, S., \& Tuszynski, J. (2002). Quantum Computation in Brain Microtubules? Decoherence and Biological Feasibility. Physical Reviews E, 65, 061901

Hameroff, S. (2006). Consciousness, Neurobiology and Quantum Mechanics: The Case for a Connection. In: The Emerging 
Physics of Consciousness, Springer (Ed.) Jack Tuszynski. Heidelberg: Springer.

Jung, C.G. (1961). Dreams, Memories, Reflections. London: Random House.

King, C. (1997). Quantum Mechanics, Chaos and the Conscious Brain. Journal of Mind and Behavior, 18, 155-170.

King, C. (2006). Quantum Cosmology and the Hard Problem of the Conscious Brain. In: J. Tuszynski (Ed.). The Emerging Physics of Consciousness. Heidelberg: Springer.

Kuwabara, T., Hsieh, J., Nakashima, K., Taira, K., \& Gage, F.H. (2004). A small modulatory dsRNA specifies the fate of adult neural stem cells. Cell, 116, 779-93.

Lein, E.S., Hawrylycz, M.J., Ao, N., Ayres, M., Bensinger, A., Bernard, A., Boe, A.F., Boguski, M.S., Brockway, K.S., Byrnes, E.J., \& other members of Allen Institute Group (2007). Genome-wide atlas of gene expression in the adult mouse brain. Nature, 445, 168-177. (Allen Brain Atlas: http://www.brain-map.org/welcome.do )

Parkinson, N., \& Brown, S. (2002). Focusing on the genetics of hearing: you ain't heard nothin' yet. Genome Biology, 3, (6), comment 2006.1-2006.6 .

6. Parsons, T.D. (2006). Auditory fidelity. Nature, 444, 10131014.

Roux, I., Safieddine, S., Nouvian, R., Grati, M., Simmler, M.C., Bahloul, A., Perfettini, I., Le Gall, M., Rostaing, P., Hamard, G., Triller, A., Avan, P., Moser, T., Petit, C. (2006). Otoferlin, Defective in a human deafness form, is essential for exocytosis at the auditory ribbon synapse. Cell, 127, 277-289. Rizzolatti, G., \& Craighero, L. (2004). The mirror neuron system. Annual Review of Neuroscience, 27, 169-92.

Roux, I., Safieddine, S., Nouvian, R., Grati, M., Simmler, M.C., Bahloul, A., Perfettini, I., Le Gall, M., Rostaing, P., Hamard, G., Triller, A., Avan, P., Moser, T., Petit, C. (2006). Otoferlin, Defective in a human deafness form, is essential for exocytosis at the auditory ribbon synapse. Cell, 127, 277-289. Rudgley, R. (1993). The Alchemy of Culture. London: British Museum Press.

Simner, J., \& Ward, J. (2006). Synesthesia: the taste of words on the tip of the tongue. Nature, 444, 438.

Su, C.Y., Luo, D.G., Terakita, A., Shichida, Y., Liao, H.W., Kazmi, M.A., Sakmar, T.P., Yau, K.W. (2006). Parietal-eye phototransduction components and their potential evolutionary implications. Science, 311, 1617-21.

Thiel, G., Lietzand, M., \& Hohl, M. (2004). How mammalian transcriptional repressors work. European Journal of Biochemistry, 271, 2855-2862.

Turin, L. (2006). The Secret of Scent: Adventures in Perfume and the Science of Smell. New York: Ecco.

Wetzel, C., Hu, J., Riethmacher, D., Benckendorff, A., Harder, L., Eilers, A., Moshourab, R., Kozlenkov, A., Labuz, D., Caspani, O., Erdmann, B., Machelska, H., Heppenstall, P.A., Lewin, G.R. (2007). A stomatin-domain protein essential for touch sensation in the mouse. Nature, 445, 206-9. 